\title{
Situación real de las cardiopatías congénitas en dos hospitales públicos del estado de Jalisco
}

\section{Current situation of congenital heart diseases in two public hospitals in the state of Jalisco}

\author{
Rocío A. Peña-Juárez ${ }^{*}$ y Miguel A. Medina-Andrade ${ }^{2}$ \\ ${ }^{1}$ Departamento de Cardiología Pediátrica, Hospital General de Occidente; ${ }^{2}$ Departamento de Cirugía Cardiovascular Pediátrica, Hospital Civil Fray \\ Antonio Alcalde. Zapopan, Jalisco, México
}

\begin{abstract}
Resumen
Introducción: Las cardiopatías congénitas representan un problema de salud pública a nivel mundial. Objetivo: Conocer la cantidad de pacientes cardiópatas atendidos en dos hospitales públicos del estado de Jalisco, así como la mortalidad y los recursos con que cuentan los hospitales participantes para la atención de estos pacientes en el período del 2015 al 2018. Se solicitó la información a la Plataforma Nacional de Transparencia y además se revisaron las bases de datos de los servicios de cardiología pediátrica y cirugía cardiovascular pediátrica de los hospitales participantes. Resultados: El hospital de segundo nivel cuenta con los recursos humanos, pero no con el material para atender a estos pacientes, por lo que no es posible ofrecer ningún tipo de tratamiento paliativo o correctivo (sólo se cierran algunos conductos arteriosos en la etapa neonatal). Se valoró a un total de 624 pacientes, de los cuales el 92.2\% correspondió a cardiopatías no críticas; la mortalidad global fue del 12\% pero en las cardiopatías críticas fue del 79.5\%. El hospital de tercer nivel cuenta con recursos humanos y material para atender a estos pacientes; en el período de estudio se realizaron 289 operaciones y la mortalidad global fue del 20.4\%. Conclusión: Las cardiopatías congénitas en el estado de Jalisco son una causa importante de mortalidad, con una incidencia elevada y una capacidad de resolución sumamente limitada, ya que los servicios de salud de Jalisco para la atención de estos pacientes son insuficientes e inadecuados. Es esencial fortalecer el sistema de salud para atender a estos pacientes.
\end{abstract}

Palabras clave: Cardiopatía congénita. Hospital. Pediatría. Mortalidad.

\begin{abstract}
Introduction: Congenital heart disease represents a public health issue worldwide. Objective: To know the number of patients with heart disease treated in two public hospitals of the State of Jalisco, as well as the mortality and resources available to participating hospitals for the care of these patients in the period from 2015 to 2018; the information was requested to the National Transparency Platform, and the database of pediatric cardiology services and pediatric cardiovascular surgery of the participating hospitals were also reviewed. Results: The second level hospital has human resources, but not the material to attend to these patients; so it is not possible to offer any type of palliative or corrective treatment. A total of 624 patients were
\end{abstract}

Correspondencia:

*Rocío A. Peña-Juárez

E-mail: alepejz@gmail.com

1405-9940/๑ 2019 Instituto Nacional de Cardiología Ignacio Chávez. Publicado por Permanyer. Este es un artículo open access bajo la licencia CC BY-NC-ND (http://creativecommons.org/licenses/by-nc-nd/4.0/).
Fecha de recepción: 08-07-2019

Fecha de aceptación: 21-10-2019
Disponible en internet: 07-02-2020 www.archivoscardiologia.com Arch Cardiol Mex. 2020;90(2):144-150 
evaluated, of which $92.2 \%$ corresponded to non-critical heart disease; overall mortality was $12 \%$ but in critical heart disease it was $79.5 \%$. The third level hospital has human and material resources to care for these patients. During the study period, 289 operations were performed and the overall mortality was $20.4 \%$. Conclusion: Congenital heart disease in the State of Jalisco is an important cause of mortality, with a high incidence and a very limited resolution capacity since the health services in the State of Jalisco for the care of these patients are insufficient and inadequate. It is essential to strengthen the health system for the care for these patients.

Key words: Congenital heart defect. Hospital. Pediatric. Mortality.

\section{Introducción}

Las cardiopatías congénitas (CC) se definen como una anomalía estructural grave del corazón o los grandes vasos intratorácicos que ocasiona una repercusión real o potencial ${ }^{1}$. Representan la forma más frecuente de defectos graves al nacimiento ${ }^{2}$. En forma general, se considera que de los 130 millones de neonatos que nacen alrededor del mundo cada año, más de un millón lo hacen con alguna $\mathrm{CC}$, lo que constituye un problema de salud pública mundial. La incidencia de CC graves se aproxima a 2.5 a 3 por cada 1,000 nacimientos ${ }^{3}$. En el 2000 se calculaba una prevalencia global de 11.9 por cada 1,000 niños ${ }^{4}$. En este país se desconoce la prevalencia real de las cardiopatías congénitas; la información de la que se dispone se basa en las tasas de mortalidad del 2015. Las CC corresponden a la segunda causa de mortalidad en los menores de un año y, en el período de 1 a 14 años, las CC ascendieron de la tercera a la segunda causa al desplazar al cáncer infantil ${ }^{5,6}$. Muchas veces, los datos acerca de la mortalidad en CC pueden interpretarse en el contexto de disponibilidad de cuidados médicos y quirúrgicos, por lo que los datos de mortalidad reflejan la evolución natural de la enfermedad ${ }^{7}$ en países con acceso limitado a los procedimientos cardioquirúrgicos. La necesidad de recursos para atender a estos pacientes es de suma importancia; de acuerdo con la propuesta de Calderón, et al., en el $2010^{5}$ se requerían alrededor de 25 centros de cirugía cardíaca para CC en México. En el estado de Jalisco existen tres grandes centros hospitalarios públicos que atienden a la población más vulnerable; sin embargo, sólo uno cuenta con el personal humano y recursos materiales para atender a estos pacientes, lo que resulta notoriamente insuficiente. En este sentido, es preciso conocer las características epidemiológicas de las cardiopatías congénitas en este estado, a fin de determinar el número necesario de cardiólogos pediatras, cirujanos cardiovasculares, intensivistas pediatras, servicios asistenciales, perfusionistas y demás recursos para afrontar de manera adecuada la atención de estos pacientes. En consecuencia, deben conocerse la magnitud y la distribución de las cardiopatías en Jalisco, de tal modo que se obtengan datos que ayuden a planificar acciones, ofrecer asistencia a los pacientes afectados y disminuir sus efectos en la mortalidad. Cabe mencionar que no se dispone de estudios estatales de base poblacional o epidemiológicos que permitan una aproximación al conocimiento de la magnitud y distribución por tipo de cardiopatía, por lo que uno de los objetivo de este estudio fue determinar la frecuencia de cardiopatías atendidas en dos de los hospitales públicos del estado de Jalisco en el período del 2015 al 2018, además de conocer la mortalidad de estos individuos en dichas instituciones y los recursos que poseen los hospitales para la atención de estos pacientes.

Más allá de la utilidad de este trabajo, el propósito principal es alertar acerca de la importancia de las cardiopatías congénitas en el estado, y de forma indirecta en muchas partes del país, y describir la realidad en este campo; asimismo, es preciso impulsar el establecimiento de acciones para proporcionar atención oportuna y de calidad a los pacientes cardiópatas en esta entidad.

\section{Material y métodos}

Se condujo un estudio descriptivo, prospectivo y multicéntrico avalado por el comité de ética del Hospital General de Occidente; se efectuó una búsqueda en las bases de datos de los servicios de cardiología pediátrica y cirugía cardiovascular pediátrica de los hospitales participantes y se solicitó además a la Plataforma Nacional de Transparencia, con los números de folios 04151419, 04151319, 04151619 y 04151519 , en el período comprendido entre 2015 y 2018, la mortalidad por cardiopatías congénitas en menores de 5 años, la cantidad de pacientes cardiópatas atendidos en dicho período y los recursos humanos y materiales disponibles para la atención de las cardiopatías en el Hospital General de Occidente de la Secretaría de Salud Jalisco (hospital de segundo nivel) y el OPD Hospital Civil de 
Tabla 1. Datos obtenidos por la Plataforma Nacional de Transparencia

\begin{tabular}{|l|l|l|l|}
\hline Hospital & $\begin{array}{l}\text { Mortalidad en menores de } \mathbf{5} \text { años } \\
\text { en el período de } \mathbf{2 0 1 5} \mathbf{a} \mathbf{2 0 1 8}\end{array}$ & $\begin{array}{l}\text { Número de pacientes cardiópatas } \\
\text { atendidos en el período de 2015 a } 2018\end{array}$ & $\begin{array}{l}\text { Recursos humanos y materiales } \\
\text { que posee cada hospital para } \\
\text { atención de estos pacientes }\end{array}$ \\
\hline $\begin{array}{l}\text { Hospital de } \\
\text { tercer nivel }\end{array}$ & No informado & 37 pacientes & $\begin{array}{l}\text { Cuenta con el personal profesional } \\
\text { y capacitado para atención médica } \\
\text { del servicio de cardiología } \\
\text { pediátrica }\end{array}$ \\
\hline $\begin{array}{l}\text { Hospital de } \\
\text { segundo nivel }\end{array}$ & 1 caso & No informado & $\begin{array}{l}\text { Cardiólogo pediatra, hemodinamista } \\
\text { pediatra, cirujano cardiovascular } \\
\text { pediatra, equipo de } \\
\text { ecocardiograma, anestesiólogo } \\
\text { pediatra, tres intensivistas } \\
\text { pediatras, ecocardiograma fijo }\end{array}$ \\
\hline
\end{tabular}

Guadalajara Fray Antonio Alcalde (hospital de tercer nivel). El análisis descriptivo se realizó para las características demográficas y clínicas de los pacientes y se midieron máximos, mínimos y porcentajes para las variables categóricas. Todas las pruebas estadísticas se llevaron a cabo con el software IBM-SPSS versión 24 (SPSS, Chicago, IL, EE.UU.).

\section{Resultados}

Se muestran los resultados en dos apartados, el primero acorde a los datos que proporciona la Plataforma Nacional de Transparencia con los números de folios 04151419, 04151319, 04151619 y 04151519 , y el segundo con base en los datos de la revisión de la base de datos de los servicios de cardiología pediátrica y cirugía cardiovascular pediátrica de los hospitales participantes.

A continuación se muestran los datos que consigna la Plataforma Nacional de Transparencia con los números de folios ya mencionados y con los distintos puntos solicitados y se enlistan en la tabla 1.

a) Mortalidad en cardiopatías en menores de cinco años en el período de 2015 a 2018:

1. Hospital de tercer nivel: no se registra la mortalidad.

2. Hospital de segundo nivel: 1 paciente.

b) Pacientes atendidos con cardiopatías menores de cinco años en el período de 2015 a 2018:

1. Hospital de tercer nivel: 37 pacientes.

2. Hospital de segundo nivel: no se registró.

c) Principales afecciones atendidas:

1. Hospital de tercer nivel: valvulopatías mitral y aórtica, malformaciones congénitas del corazón específicas e inespecíficas.

2. Hospital de segundo nivel: comunicación interauricular, comunicación interventricular, conducto arterioso permeable, arritmias, enfermedad de Kawasaki, hipertensión pulmonar.

d) Recursos humanos y materiales con que cuenta cada hospital para atender a estos pacientes.

1. Hospital de tercer nivel: existe personal profesional y capacitado para suministrar atención médica del servicio de cardiología pediátrica.

2. Hospital de segundo nivel: un cardiólogo pediatra, un hemodinamista pediatra, un cirujano cardiovascular pediatra, un anestesiólogo pediatra y tres intensivistas pediatras. Un ecocardiograma fijo.

Los datos obtenidos, según la revisión de expedientes de los servicios de cardiología pediátrica y cirugía cardiovascular pediátrica de los hospitales participantes, son los siguientes:

a) Hospital de segundo nivel. En el apartado de los recursos de que dispone para la atención de estos pacientes no se identificaron diferencias respecto de lo notificado en la Plataforma Nacional de Transparencia.

En cuanto a la cantidad de pacientes con cardiopatías en el período de 2015 a 2018 se detectó un total de 624 pacientes con algún tipo de cardiopatía, de los cuales el $92.1 \%$ ( $n=575)$ corresponde a cardiopatías no críticas y el resto $(n=49)$ a trastornos críticos. Su población principal atendida corresponde a pacientes en el período neonatal, por lo que el $56.81 \%$ de las cardiopatías reconocidas corresponde a conducto arterioso permeable que, en caso de requerir cierre quirúrgico, lo realiza el servicio de cirugía pediátrica.

Este hospital, a pesar de contar con la mayor parte de los recursos humanos para atender a los pacientes con CC, no dispone del material para practicar algún tipo de intervención, por lo que los pacientes que necesitan alguna atención deben derivarse a otra institución; el $50.2 \%$ de la población $(n=313)$ requería traslado a un tercer nivel; sin embargo, sólo el $25.5 \%$ de estos enfermos pudo enviarse a un tercer nivel; 
Tabla 2. Datos obtenidos de los expedientes clínicos del servicio de cardiología del hospital de segundo nivel de la Secretaría de Salud Jalisco

\begin{tabular}{|l|l|l|l|}
\hline Hospital & $\begin{array}{l}\text { Mortalidad por cardiopatías en menores } \\
\text { de } \mathbf{5} \text { años en el período de } \mathbf{2 0 1 5} \text { a } \mathbf{2 0 1 8}\end{array}$ & $\begin{array}{l}\text { Pacientes atendidos por cardiopatías } \\
\text { en el período de 2015 al 2018 }\end{array}$ & $\begin{array}{l}\text { Recursos humanos y } \\
\text { materiales disponibles }\end{array}$ \\
\hline $\begin{array}{l}\text { Hospital de } \\
\text { segundo nivel }\end{array}$ & $\begin{array}{l}\text { Global, 12\% } \\
\text { Cardiopatías críticas, 79.5\% } \\
\text { Cardiopatías no críticas, 6\% }\end{array}$ & $\begin{array}{l}\text { 624 pacientes } \\
\text { Cardiopatías críticas } 49(\mathbf{7 . 8 \% )} \\
\text { Cardiopatías no críticas }(92.2 \%)\end{array}$ & $\begin{array}{l}\text { Cardiólogo pediatra, } \\
\text { hemodinamista pediatra, } \\
\text { cirujano cardiovascular, } \\
\text { anestesista pediatra y dos } \\
\text { intensivistas pediatras } \\
\text { Ecocardiograma portátil }\end{array}$ \\
\hline
\end{tabular}

Tabla 3. Datos obtenidos de la revisión de expedientes del hospital de tercer nivel de la Secretaría de Salud Jalisco

\begin{tabular}{|c|c|c|c|}
\hline Hospital & $\begin{array}{l}\text { Mortalidad por cardiopatías en menores } \\
\text { de } 5 \text { años en el período de } 2015 \text { a } 2018\end{array}$ & $\begin{array}{l}\text { Pacientes atendidos por cardiopatías } \\
\text { en el período de } 2015 \text { a } 2018\end{array}$ & $\begin{array}{l}\text { Recursos humanos y } \\
\text { materiales con que cuenta }\end{array}$ \\
\hline $\begin{array}{l}\text { Hospital de } \\
\text { tercer nivel }\end{array}$ & Global, $20.4 \%$ & 289 operaciones & $\begin{array}{l}\text { Dos cardiólogos pediatras, un } \\
\text { cirujano cardiovascular } \\
\text { pediatra, bomba de circulación } \\
\text { extracorpórea, intensivistas } \\
\text { pediatras, dos perfusionistas, } \\
\text { anestesista pediatra, un } \\
\text { ecocardiograma portátil }\end{array}$ \\
\hline
\end{tabular}

cabe mencionar que muchos de estos pacientes se remitieron por la consulta externa a otro hospital. En relación con las cardiopatías críticas de los 49 casos, 39 no pudieron trasladarse (79.59\%) y de los 10 casos que se trasladaron sólo dos se recibieron en un hospital de la Secretaría de Salud (en el resto, los familiares consiguieron algún tipo de seguridad social).

En cuanto a la mortalidad, se identificó una mortalidad global del $12 \%$ de la población; debe señalarse que los 39 pacientes que sufrían algún tipo de cardiopatía crítica y que no se trasladaron a otra unidad fallecieron (el resto de la mortalidad corresponde a conductos arteriosos; Tabla 2).

b) Hospital de tercer nivel

Con base en los datos obtenidos en la revisión de los expedientes del servicio de cirugía cardiovascular pediátrica se encontró que entre 2015 y 2018 se practicó un total de 289 operaciones; las cardiopatías que se atendieron fueron conducto arterioso permeable en $36.5 \%(n=105)$, comunicación interventricular en $25 \%$ ( $n=72)$, comunicación interauricular en $16 \%(n=45)$, coartación aórtica en $14 \%(n=41)$, tetralogía de Fallot en $6 \%(n=19)$, doble salida del ventrículo derecho en $1 \%(n=3)$ y atresia tricuspídea en $1.38 \%(n=4)$.

La mortalidad informada fue de 59 pacientes $(20.4 \%)$, de los cuales el $80 \%(n=47)$ falleció en terapia intensiva pediátrica.

Los recursos disponibles incluyen dos cardiólogos pediatras, un cirujano cardiovascular pediatra, intensivistas pediatras, perfusionistas y bomba de circulación extracorpórea (Tabla 3).

\section{Discusión}

Muchas enfermedades cardiovasculares no pueden prevenirse y evolucionan de manera independiente en una etapa o edad posteriores. Las CC ocurren en cerca de 1 por cada 1,000 nacimientos y poseen un componente heterogéneo que influye en la incidencia y evolución de la enfermedad. Incluso en condiciones óptimas, es decir, pobreza erradicada, práctica de ejercicio, alimentación saludable, y sin beber ni fumar, la enfermedad cardiovascular aún ocurriría en cierta medida $^{8}$. La incidencia de CC graves se aproxima a 2.5 a 3 por cada 1,000 nacimientos. Las formas moderadas de las CC son de casi 3 por cada 1,000 nacimientos, pero otros 13 por cada 1,000 nacimientos desarrollan $\mathrm{CC}$ leves que al final exigirán cuidados cardiológicos ${ }^{3}$. En este país no existen datos que orienten acerca de la prevalencia real de las cardiopatías congénitas; sin embargo, puede considerarse un promedio teórico, derivado de la información mundial: 8 por 1,000 nacidos vivos. Al relacionar esta cifra con la tasa de natalidad anual en México $(250,0000)$, se puede inferir que cada año nacen alrededor de 18000 a 21000 niños con algún tipo de malformación cardíaca ${ }^{5}$. En el año 2017 en el estado de Jalisco nacieron 151,950 niños ${ }^{6}$; si se toma en cuenta la tasa de prevalencia ya mencionada, en Jalisco nacieron en 2017 alrededor de 1,216 niños con alguna cardiopatía congénita, de los cuales cerca de la mitad desarrollará una cardiopatía clínicamente significativa ${ }^{9}$ y requerirá algún tipo de intervención; esto significa que en Jalisco tan sólo en el 2017 un 
poco más de 600 niños necesitó atención cardiovascular urgente, pero en un hospital de segundo nivel no es posible ofrecer ningún tipo de tratamiento y esa cantidad de pacientes se valoró en los tres años del estudio y, peor aún, en el hospital de tercer nivel se realizó la mitad de las intervenciones requeridas en los tres años del estudio en comparación con lo que era necesario atender en un solo año, esto es, menos de un tercio de la población tuvo acceso al tratamiento cardiológico y la mayoría de los casos fue subóptimo.

En términos históricos, la mayoría de los pacientes con CC muere en etapas tempranas de la infancia. Sin embargo, en las últimas cuatro décadas se han conseguido avances extraordinarios en este campo. En realidad, los últimos estudios de prevalencia de la población indican que ahora el número de adultos es casi el mismo que el número de niños con defectos graves $^{10}$. En los países con altos ingresos, la mortalidad de las CC ha disminuido en grado notable, pero no en los de ingresos medios o bajos. La diferencia de mortalidad entre países industrializados y el tercer mundo es notoria, de $3 \%$ a $7 \%$ y hasta $20 \%$, respectivamente. Sin embargo, es probable que la mortalidad de CC esté subestimada en países tercermundistas por el escaso acceso al diagnóstico y la gran mayoría de los estudios sólo notifica los casos de pacientes de centros de tercer nivel ${ }^{5}$, como en el caso de Jalisco. La mortalidad registrada aquí es sumamente alta; en el hospital de segundo nivel, el $80 \%$ de los pacientes con cardiopatía crítica fallece sin poder someterse a ningún tipo de procedimiento paliativo y mucho menos correctivo; además, se registra hasta el $6 \%$ de mortalidad en las cardiopatías no críticas, que corresponden a conductos arteriosos en pacientes neonatales. En el hospital de tercer nivel, la mortalidad quirúrgica es de un poco más del $20 \%$. No obstante, los datos acerca de mortalidad en CC pueden interpretarse muchas veces en el contexto de disponibilidad de cuidados médicos y quirúrgi$\cos ^{5}$; existen centros hospitalarios en la Ciudad de México que registran una mortalidad en cardiopatías congénitas del 2.9\% ${ }^{11}$. ¿Por qué existe tanta diferencia entre una entidad y otra? La elevada mortalidad en cardiopatías congénitas en Jalisco es multifactorial y las diferencias respecto de los hospitales de la Ciudad de México podrían deberse quizá a a) la falta de acceso a centros especializados y referencias tardías, b) altos costos para la atención de estos pacientes en un sistema de salud burocrático y con fondos insuficiente; c) ausencia de unidades multidisciplinarias y especializadas para pacientes con $\mathrm{CC}$, y d) falta de médicos y enfermeras capacitados para la atención de estos pacientes ${ }^{11}$.

En lo que corresponde a los recursos hospitalarios, uno de los datos más importantes que deben valorarse en estos pacientes es el alcance del tratamiento y el efecto en los resultados para las CC; la atención médica y quirúrgica de las $\mathrm{CC}$ requiere muchos recursos. En realidad, a menudo es complejo establecer un diagnóstico preciso y para hacerlo se necesitan modalidades diagnósticas costosas, además de especialistas médicos altamente capacitados. El tratamiento quirúrgico de la CC también consume recursos considerables, infraestructura y equipos complejos, y profesionales altamente certificados. Obviamente es muy costoso ${ }^{7}$. En cuanto a la infraestructura, según la propuesta de Calderón, et al. del $2010^{5}$, basada a las recomendaciones establecidas por el Comité de Cardiopatías Congénitas de la Asociación Europea de Cirujanos Cardiotorácicos, y en relación con una población mexicana calculada de 103 millones de habitantes, se requieren alrededor de 25 centros de cirugía cardíaca para cardiopatías congénitas. Por otra parte, en México nacen al año cerca de dos millones de niños. Si se consideran los criterios de la Academia Americana de Pediatría, es decir, un centro médico-quirúrgico por cada 30,000 nacidos vivos, los requerimientos hospitalarios serían de 66 unidades de cirugía cardíaca. Jalisco cuenta con 8.2 millones de habitantes y por tanto se requieren dos centros cardiovasculares quirúrgicos, de acuerdo con las guías del Comité de Cardiopatías Congénitas de la Asociación Europea de Cirujanos Cardiotorácicos y cinco centros cardiovasculares según las guías de la Academia Americana de Pediatría. Sin embargo, en este estado sólo existen dos centros que realizan operaciones en cardiopatías congénitas, uno de seguridad social y un hospital de tercer nivel de la Secretaría de Salud que atiende a la población sin seguridad social y que muchas veces es la más vulnerable. Con base en los protocolos del Comité de Cardiopatías Congénitas de la Asociación Europea de Cirujanos Cardiotorácicos, para considerar rentable un centro que practica intervenciones cardíacas se requiere lo siguiente ${ }^{12}$ :

1. El número intervenido por año debe ser al menos de 250 y mayor de 100 casos por año en recién nacidos y menores de un año.

2. Cada cirujano debe efectuar un mínimo de tres intervenciones por semana y 126 por año.

3. En caso de unidades con menor volumen (menos de 250 pacientes al año) se las puede considerar como 
hospitales funcionales si los resultados son similares a los centros que atienden a mayor volumen.

La cantidad de pacientes operados en el hospital de tercer nivel es de 289 en tres años, es decir, dicho hospital no es rentable; sin embargo, no puede alcanzarse ese objetivo porque no cuenta con un equipo multidisciplinario (cardiólogos pediatras, cirujanos cardiovasculares pediatras, intensivistas cardiovasculares pediatras, enfermeras intensivistas cardiovasculares, perfusionistas, anestesiólogo cardiovascular pediatra, trabajadora social, psicología, etc.) y, dado que se trata de un hospital público, tiene notables limitaciones financieras y una organización altamente burocrática que le impide establecer los sistemas de control de alta calidad. Paradójicamente, el hospital de segundo nivel posee la mayor parte de los recursos humanos necesarios para atender a los pacientes con CC y sin embargo no cuenta con el apoyo del gobierno para obtener recursos materiales para la atención de estos enfermos.

Otro punto importante que debe tomarse en cuenta, en la elevada mortalidad de los pacientes con CC en el estado de Jalisco, es el inadecuado sistema de referencia, ya que en muchos de los casos no es posible su traslado a una unidad que practique procedimientos para salvar la vida, al margen de que se trate de una cardiopatía crítica que exija atención inmediata. Esto es consecuencia del volumen de pacientes y los recursos insuficientes de los hospitales públicos, lo cual evidentemente contribuye al incremento de la mortalidad. Como se mencionó con anterioridad, se requieren equipos multidisciplinarios que atiendan a los pacientes con cardiopatías congénitas para obtener mejores resultados; no obstante, en muchas ocasiones los pacientes reciben atención primaria de médicos escasamente capacitados en cardiología pediátrica y con frecuencia derivan y detectan a los pacientes en una fase muy tardía. En suma, muchas de estas muertes ocurren debido a la mala calidad de la atención por los recursos hospitalarios ineficientes y circunstancias sociales adversas.

Por último, cabe mencionar la gran discrepancia entre la información obtenida a través del sistema de transparencia y la información real de los hospitales; es por tanto muy importante mejorar los sistemas de estadística del estado de Jalisco para consignar datos reales de los pacientes atendidos en este estado.

A pesar de que los equipos de atención cardiológica (cardiólogos pediatras, cirujanos cardiovasculares pediatras, intensivistas pediatras, enfermeras, perfusionistas) que atienden en los hospitales de Jalisco intentan proporcionar una atención oportuna y de calidad a estos pacientes, esto no es suficiente por las grandes limitaciones; aún queda mucho por hacer para garantizar que todos los niños nacidos en el estado con anomalías cardíacas reciban cuidados médicos y quirúrgicos apropiados y oportunos.

\section{Conclusión}

Las cardiopatías congénitas en el estado de Jalisco son una causa importante de mortalidad, con una incidencia elevada y una capacidad de resolución sumamente limitada, ya que los servicios de salud en el estado de Jalisco para la atención de estos pacientes son sumamente insuficientes e inadecuados.

Según el artículo 4 de la Constitución Mexicana, "Los niños y las niñas tienen derecho a la satisfacción de sus necesidades de alimentación, salud, educación y sano esparcimiento para su desarrollo integral. El Estado otorgará facilidades a los particulares para que se coadyuven al cumplimiento de los derechos de la niñez". La salud es un derecho humano básico. Todos los niños que nacen con CC merecen recibir un tratamiento y tener los accesos a intervenciones capaces de salvar su vida y éstos no deben reservarse para aquéllos nacidos en áreas específicas del país. Ningún niño con $\mathrm{CC}$ debe morir por falta de recursos.

Para superar esta gran disparidad en el acceso a los servicios de cirugía cardíaca es esencial fortalecer el sistema de salud para atención de estos pacientes.

\section{Financiamiento}

Ninguno.

\section{Conflicto de intereses}

Los autores declaran no tener conflicto de intereses alguno.

\section{Responsabilidades éticas}

Protección de personas y animales. Los autores declaran que para esta investigación no se han realizado experimentos en seres humanos ni en animales.

Confidencialidad de los datos. Los autores declaran que han seguido los protocolos de su centro de trabajo sobre la publicación de datos de pacientes. 
Derecho a la privacidad y consentimiento informado. Los autores declaran que en este artículo no aparecen datos de pacientes.

\section{Bibliografía}

1. Mitchell SC, Korones SB, Berendes W. Congenital heart disease in 56,109 births incidence and natural history. Circulation 1971;43:323-332.

2. Khairy $\mathrm{P}$, lonescu-lttu R, Mackie AS, Abrahamowicz M, Pilote L, Marelli AJ. Changing mortality in congenital heart disease. J Am Coll Cardiol 2010;56(14):1149-1157.

3. Hoffman JI, Kaplan S. The incidence of congenital heart disease. J Am Coll Cardiol 2002;39(12):1890-900.

4. Marelli Aj, Mackie AS, Ionescu-Ittu R, Rahme E, Pilote L. Congenital heart disease in the general population: changing prevalence and age distribution. Circulation 2007;115(2):163-172.

5. Calderón-Colmenero J, Cervantes-Salazar JL, Curi-Curi PJ, Ramírez- Marroquín S. Problemática de las cardiopatías congénitas en México. Propuesta de regionalización. Arch Cardiol Mex 2010;80(2):133-140.
6. INEGI, Nacimientos registrados, por entidad federativa período y sexo 2015. https://www.inegi.org.mx/programas/natalidad/default.html\#Tabulados, revisado 03/07/2019

7. Bernier PL, Stefanescu A, Samoukovic G, Tchervenkov Cl. The challenge of congenital heart disease worldwide: epidemiologic and demographic facts. Semin Thorac Cardiovasc Surg Pediatr Card Surg Annu 2010;13(1):26-34

8. Vervooot D. The neglected component of cardiovascular care. Glob Heart 2019;18:1-3.

9. Gelb BD. Genetic basis of congenital heart disease. Curr Opin Cardiol 2004;19(2):110-5

10. Khairy $\mathrm{P}$, lonescu-Ittu R, Mackie AS, Abrahamowicz M, Pilote L, MareIli AJ. Changing mortality in congenital heart disease. J Am Coll Cardiol 2010;56(14):1149-57.

11. Palacios-Macedo A, Mery CM, Cabrera AG, Basteros P, Tamariz-Cruz O, Diliz-Nava $\mathrm{H}$, et al. A novel private-public hybrid model for treatment of congenital heart disease in Mexico. World J Pediatr Congenit Heart Surg 201910(2):206-213.

12. Daenen W, Lacourt-Gayet $F$, Aberg T. Optimal structure of a congenital heart surgery department in Europe by EACTS congenital heart disease committee. Eur J Cardiothorac Surg 2003;24(3):334-51. 